\title{
Combinação Convexa de Filtros Adaptativos Set-Membership
}

\author{
Jonnathan F. Partamian e Vítor H. Nascimento
}

\begin{abstract}
Resumo - Neste artigo propomos uma combinação convexa de filtros Set-Membership NLMS (SM-NLMS). Iniciamos analisando os efeitos do uso de diferentes parâmetros no filtro Set-Membership simples para então verificar as possíveis características a combinar e suas aplicações.

São apresentadas combinações utilizando-se filtros de diferentes comprimentos e com diferentes valores de limiar de erro, $\gamma$. É mostrado que usar diferentes comprimentos resulta em um filtro com maior velocidade de convergência, enquanto que combinar filtros com diferentes $\gamma$ resulta em um filtro SM menos sensível a variações na SNR, o que também tem a vantagem de aumentar a robustez a erros de detectores de double-talk.

Por fim é apresentado um algoritmo de cálculo eficiente para combinações de filtros SM, que tira proveito da sua característica de atualização esparsa de seus coeficientes. Deste algoritmo obtemos duas variações, uma delas bastante eficaz no cálculo de combinações de filtros SM de comprimentos diferentes.

Palavras-chave - Filtros adaptativos, combinação convexa, Set-Membership, implementações eficientes.
\end{abstract}

Abstract - This paper proposes a Set-Membership NLMS (SMNLMS) filter convex combination. We began analyzing the effects of the use of different parameters in simple SetMembership filter for then to verify the possible characteristics to combine and their applications. It is shown that to use different lengths results in a filter with larger convergence speed, while combining filters with different error bounds $\gamma$ results in a SM filter less sensitive to SNR variations, what also has the advantage of increasing the robustness to double-talk detectors errors.

At the end is presented an algorithm of efficient calculation for SM filters combinations that take advantage of its characteristic of scattered updating of its coefficients. Of this algorithm we obtain two variations, one of them quite effective in the calculation of SM filters combinations of different lengths.

Keywords - Adaptive filters, convex combination, SetMembership, efficient implementations.

\section{INTRODUÇÃO}

$\mathrm{E}$ M sistemas viva-voz, a parcela do som emitido pelo altofalante que alcança o microfone é chamada de eco acústico. Uma das formas de se atenuar este efeito, que gera o incômodo do interlocutor distante (far-end speaker) ouvir o eco de sua própria voz, é através da técnica de filtragem adaptativa. Neste método, o filtro adaptativo tenta modelar a resposta impulsiva $\boldsymbol{w}(n)$ do caminho acústico entre o altofalante (AF) e o microfone, gerando a partir do sinal que vai para o AF um eco estimado, o qual é subtraído do sinal captado pelo microfone.
O processo de adaptação do filtro depende de que o sinal captado pelo microfone seja o mais próximo possível do eco que se deseja cancelar, ou seja, que este tenha o mínimo possível de sons adicionais como ruídos do ambiente e voz do interlocutor local (near-end speaker). Para isso, a adaptação é controlada de forma a ocorrer apenas quando o interlocutor distante estiver falando, motivo pelo qual é necessário um detector de fala simultânea (double-talk detector, DTD), responsável por parar a adaptação do filtro quando ambos os interlocutores estiverem falando ao mesmo tempo.

Atualmente os principais desafios nesta técnica de cancelamento de eco acústico (AEC) são quanto à velocidade com que o filtro é capaz de se adaptar às mudanças de $\boldsymbol{w}(n)$ (tracking e velocidade de convergência), a qualidade do DTD e a complexidade computacional envolvida, dado o grande número de coeficientes necessários para o filtro.

Uma das técnicas de filtragem adaptativa utilizada em AEC é o Set-Membership (SM) [1], que possui duas características importantes: tamanho de passo otimizado e adaptação seletiva. O tamanho de passo otimizado leva a uma velocidade de convergência maior, enquanto que a adaptação seletiva (baseada em um limiar de erro) implica um menor desajuste, especialmente em casos onde o ruído adicional (ao sinal do eco, no caso) é grande. A adaptação seletiva também faz com que o filtro demande uma menor carga computacional, já que seus parâmetros não são atualizados a todo tempo.

A principal dificuldade no uso do SM é a definição do seu limiar de erro $\gamma$ [2], parâmetro crítico para este tipo de filtro: um limiar muito alto diminui a taxa de atualização, mas faz com que o filtro adapte menos do que o necessário, levando a um desajuste exagerado e reduzindo a capacidade de tracking; um limiar muito baixo faz com que o filtro seja atualizado mesmo quando esse ajuste não é favorável (o que aumenta o desajuste), além de aumentar a carga computacional devido ao número de adaptações exagerado.

Visando resolver o problema de definição do limiar de erro no SM, bem como o da probabilidade de falha na detecção de double-talk, além das questões relativas à complexidade computacional, estudaremos neste artigo a combinação convexa de filtros SM (SMF) com diferentes parâmetros. Nessa técnica (descrita na Seção II) combinam-se as saídas de dois ou mais filtros (cada um operando de forma independente), visando reunir suas diferentes qualidades em um só filtro.

Assim, primeiramente estudaremos na Seção III os efeitos da utilização de diferentes parâmetros no SM-NLMS, para 
verificarmos quais diferentes características poderiam ser combinadas. Então, na Seção IV, avaliaremos a combinação convexa de dois SMF com parâmetros diferentes e suas aplicações no AEC, além de um método para cálculo eficiente deste tipo de combinação. Por fim apresentaremos as conclusões na Seção V.

\section{DeSCRIÇÃo dOS AlgoritMos}

A Fig. 1 mostra um esquema típico de cancelamento de eco acústico, onde $u(n)$ é o sinal captado pelo microfone distante (far end speaker), $\boldsymbol{w}(n)$ é a resposta impulsiva de ordem $\mathrm{M}$ do caminho acústico entre o alto-falante e o microfone, $y^{\prime}(n)$ é o eco acústico, $v(n)$ é a soma do sinal de voz e o ruído do ambiente, $\quad d(n)=y^{\prime}(n)+v(n)$ é sinal captado pelo microfone local, $\widehat{\boldsymbol{w}}(n)$ é a resposta impulsiva de ordem $N \leq M$ do filtro adaptativo, que tenta reproduzir $\boldsymbol{w}(n), y(n)$ é o eco estimado e $e(n)$ é o sinal enviado ao alto-falante distante.

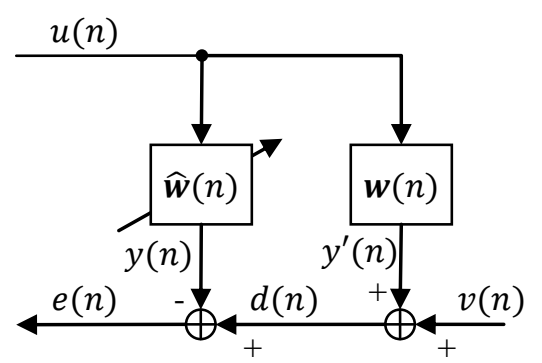

Fig. 1. Esquema típico de cancelamento de eco acústico.

\section{A. Combinação Convexa}

$\mathrm{Na}$ técnica de combinação convexa, dois ou mais filtros são adaptados separadamente, tendo suas saídas combinadas de acordo com um parâmetro de mistura que é adaptado de modo a reduzir o erro da mistura, juntando no filtro combinado as melhores características daqueles que o compõe.

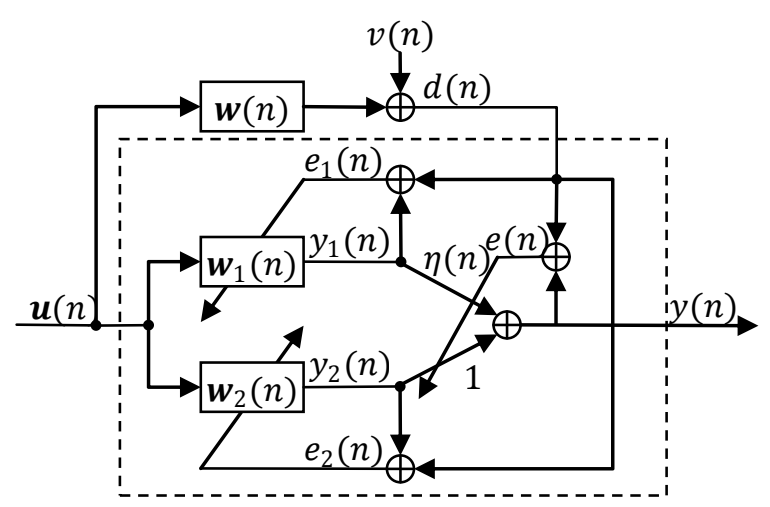

Fig. 2. Combinação convexa de dois filtros transversais.

Utilizaremos aqui o mesmo esquema de combinação de [3], conforme a Fig. 2, cuja saída geral é

$$
y(n)=\eta(n) y_{1}(n)+[1-\eta(n)] y_{2}(n)
$$

onde $y_{i}(n)=\boldsymbol{u}^{T}(n) \boldsymbol{w}_{i}(n), i=1,2$ são as saídas dos filtros transversais, $\boldsymbol{w}_{i}(n)$ a resposta impulsiva de cada um deles e $\eta(n)$ o parâmetro de mistura, que é dado pela seguinte função sigmoidal (sgm):

$$
\eta(n)=\operatorname{sgm}[a(n-1)]=\frac{1}{1+e^{-a(n-1)}}
$$

sendo $a(n)$ uma variável auxiliar calculada por:

$$
\begin{aligned}
& a(n)=a(n-1) \\
& +\mu_{a} e(n)\left[y_{1}(n)-y_{2}(n)\right] \eta(n)[1-\eta(n)]
\end{aligned}
$$

com $\mu_{a}$ sendo o passo de adaptação de (3).

Como descrito em [4], o fator $\eta(n)[1-\eta(n)]$ reduz a velocidade de adaptação (e conseqüentemente o ruído do gradiente) próximo a $\eta(n)=0$ e $\eta(n)=1$, mas também pode fazer com que a adaptação de $a(n)$ pare próximo a esses limites (já que $\eta(n)[1-\eta(n)]$ resultaria em um número próximo de zero). Por essa razão, $|a(n)|$ deve ser limitado. Como em [4] e [5], usaremos $|a(n)| \leq 4$.

\section{B. Set-Membership}

A filtragem set-membership (SMF) apresenta um conceito de adaptação seletiva, em que os parâmetros do filtro só são atualizados se o erro de estimação for maior que um certo valor.

Como mencionado em [2], essa classe de filtros adaptativos pretende oferecer menor complexidade computacional e menor desajuste, além de possivelmente uma convergência mais rápida. A menor complexidade computacional seria devida às atualizações esparsas dos coeficientes; o menor desajuste segue da hipótese do filtro não ser adaptado quando o ruído adicional $v(n)$ constituir uma parcela importante do erro de estimação $e(n)$; a maior velocidade de convergência estaria ligada ao passo de adaptação variável, relativo à proporção entre o erro de estimação e o limiar de erro do SMF.

Aqui utilizaremos o SM-NLMS, que atualiza seus coeficientes segundo a expressão abaixo:

$$
\boldsymbol{w}(n+1)=\boldsymbol{w}(n)+\frac{\alpha(n)}{\|\boldsymbol{u}(n)\|} \boldsymbol{u}(n) e^{*}(n)
$$

em que $\alpha(n)$ é o passo de adaptação, dado por:

$$
\alpha(n)=\begin{array}{cc}
\mu_{S M}\left(1-\frac{\gamma}{|e(n)|}\right) & \text { se }|e(n)|>\gamma \\
0 & \text { caso contrário }
\end{array}
$$

$\gamma$ é o parâmetro que define o limiar a partir do qual deve-se ou não ajustar o vetor $\boldsymbol{w}$ e $\mu_{S M}$ é uma constante que inserimos para ajustar o tamanho do passo. 


\section{INFLUÊNCIA DE DIFERENTES PARÂMETROS DO SMF NO CANCELAMENTO DE ECO ACÚSTICO}

Nesta seção analisaremos a influência de diferentes valores de $\mu_{S M}, \gamma$ e $\mathrm{N}$ no SMF aplicado ao AEC.

Em todas as simulações foram utilizados 8 trechos de fala com 8 segundos de duração (amostrados a $8 \mathrm{kHz}$ ), aplicados à resposta impulsiva obtida em um automóvel, de $M=256$ cedida por Magno T. M. Silva e usada em [6]. Nessas simulações também foi adicionado um ruído branco gaussiano com potência $\sigma_{v}^{2}$ proporcional à potência $\sigma_{y^{\prime}}^{2}$ do sinal $y^{\prime}(n)$, com diversas SNR. A SNR é dada por: $S N R=10 \log \frac{\sigma_{y^{\prime}}^{2}}{\sigma_{v}^{2}} d B$

Os resultados são expressos em termos do ERLE (echo return loss enhancement), que é uma medida da atenuação do eco obtida pelo AEC, dada por [7]:

$$
E R L E=10 \log \frac{E\left[d^{2}(n)\right]}{E[d(n)-y(n)]} d B
$$

Para facilitar a visualização dos dados, nas figuras apresentadas a seguir foi aplicada uma média móvel de 100 amostras ao ERLE.

\section{A. Diferentes comprimentos $N$}

Para avaliar a influência da relação $N / M$ no SM aplicado ao AEC, foram feitas simulações com os seguintes valores de $\mathrm{N}$ : 256, 128, 64 e 32, mantendo-se todos os demais parâmetros fixos: $S N R=20 d B, \gamma=\sqrt{5} \sigma_{v}$ e $\mu_{S M}=1$ (conforme [8] e [2]).

Essas simulações mostraram que a redução do comprimento do filtro (até certo limite), ainda que sem o aumento do tamanho do passo $\mu_{S M}$, leva a um aumento na taxa de convergência. Este resultado coaduna com a análise feita em [9] para filtros LMS, onde é demonstrado que nessa classe de filtros, para sinais de entrada muito correlacionados (como no caso do sinal de voz), a taxa de convergência aumenta com a diminuição do comprimento do filtro (veja também [10]).

Essa característica poderia ser aproveitada combinando-se um filtro de comprimento menor com outro de comprimento maior, unindo assim a maior velocidade de convergência do primeiro ao menor desajuste do segundo.

\section{B. Diferentes passos de adaptação $\mu_{S M}$}

Sabe-se que o NLMS converge mais rápido com passo 1 [11], com sua velocidade de convergência caindo até chegar em 2 quando passa a se tornar instável. Filtros SM-NLMS já possuem um passo de adaptação otimizado, pois quando $|e(n)| \gg \gamma$, seu passo de adaptação é 1 (valor ótimo do NLMS), diminuindo progressivamente à medida que $|e(n)|$ se aproxima de $\gamma$ até ficar em zero para $|e(n)|<\gamma$, o que evita o problema do erro em excesso em razão do tamanho do passo.

Assim sendo, conclui-se que o tamanho do passo $\mu_{S M}=1$ usado no SM-NLMS já é ótimo, não sendo útil fazer uso de passos diferentes.

\section{Diferentes limiares de erro $\gamma$}

Sabe-se que o parâmetro $\gamma$ tem uma importância fundamental no SMF, já que o desajuste e a taxa de atualização do filtro dependem criticamente de sua especificação correta ( [2], [1]).

Para avaliar qual é o melhor valor de $\gamma$ e também quão crítica é a sua escolha, foram feitas simulações com diversos valores de $\gamma$, proporcionais a $\gamma^{\prime}=\sqrt{5} \sigma_{v}$ (que é um valor típico na literatura, e.g. [8], [2], [1]), mantendo-se todos os demais parâmetros fixos: $S N R=20 d B, N=256$ e $\mu_{S M}=1$. O resultado dessas simulações é apresentado nas figuras Fig. 3 e Fig. 4.

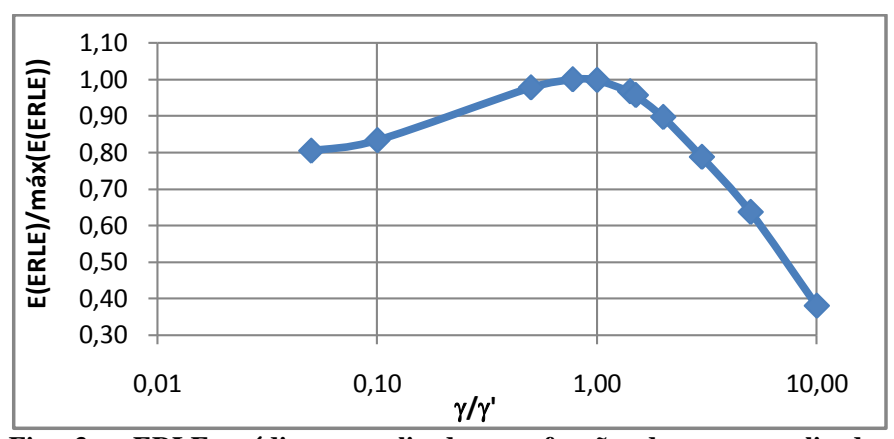

Fig. 3. ERLE médio normalizado em função do $\gamma$ normalizado. SNR=20dB.

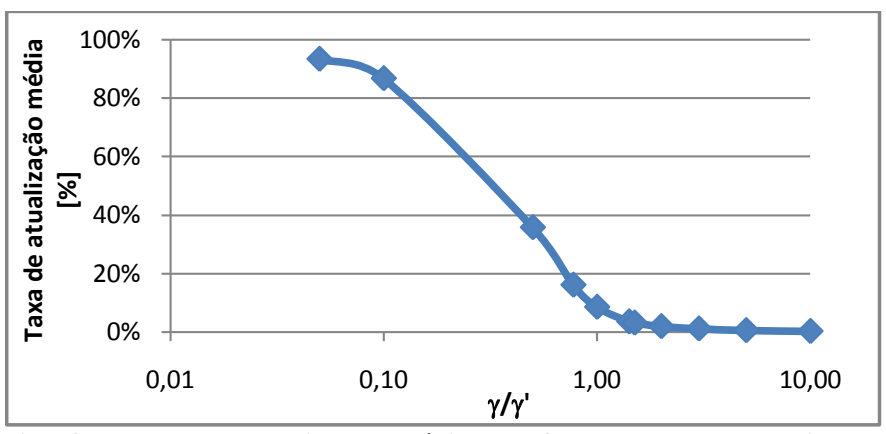

Fig. 4. Taxa de atualização média em função do $\gamma$ normalizado. SNR=20dB.

Das figuras Fig. 3 e Fig. 4 pode-se concluir que de fato o SM apresenta um maior ERLE médio para $\gamma$ com valores próximos a $\sqrt{5} \sigma_{r}$, com taxa de atualização inferior a $10 \%$. Para $\gamma$ maiores, o ERLE decai rapidamente enquanto a taxa de atualização fica ainda menor, ambos os efeitos justificados pela tolerância a um erro maior. Valores menores de $\gamma$ fazem a taxa de atualização aumentar rapidamente, enquanto que o ERLE decai numa proporção menor, tendendo ao comportamento do NLMS para $\gamma=0$, onde obtivemos um ERLE normalizado de 0,77 nas mesmas condições.

Estes resultados podem ser estendidos para a análise do caso de diferentes SNR para um mesmo $\gamma$, por exemplo, se o $\gamma$ tivesse sido mantido constante e a SNR reduzida de $20 \mathrm{~dB}$, a taxa de atualização seria a mesma do caso $\gamma / \gamma^{\prime}=10$, com um ERLE inferior ao "ótimo" na mesma proporção deste caso. A equação geral para este cálculo fica:

$$
S N R^{\prime}-S N R=20 \log \frac{\gamma}{\gamma^{\prime}} d B
$$


É importante ressaltar que, apesar de aqui o $\gamma$ ter um valor absoluto independente da amplitude de $u(n)$, caso se saiba a SNR a priori, pode-se fazer um algoritmo que calcule o $\gamma(n)$ em função de uma dada SNR.

Destes resultados podemos concluir que, como esperado, se for conhecida de antemão a SNR, pode-se determinar com facilidade o melhor $\gamma$ para o filtro SM, e este terá um desempenho superior ao NLMS tanto com relação ao ERLE com quanto à carga computacional. Porém, se a SNR não for conhecida ou variar em uma faixa relativamente grande, o SM pode ter seu desempenho seriamente afetado, seja com relação à carga computacional, seja quanto ao ERLE.

Visando resolver estes problemas foram desenvolvidos alguns algoritmos de $\gamma$ variável, por exemplo: [12] propõe um $\gamma(\boldsymbol{w})$ cujo valor é ajustado em função da norma de $\boldsymbol{w}$, para um problema de equalização, em que a potência do ruído também é função de $\boldsymbol{w}$; [2] apresenta um algoritmo em que o $\gamma$ é ajustado de modo a se obter uma taxa de atualização constante no SMF. Ambos os algoritmos ainda necessitam de alguma informação prévia do ruído.

Uma alternativa na solução do problema de definição do valor de $\gamma$ é a combinação convexa de filtros SM com diferentes $\gamma$. Com isso a combinação se encarregaria de escolher a saída do SM com o $\gamma$ mais apropriado, o que será mais bem discutido na Seção IV.

\section{CombinaÇão ConveXa do SMF}

Como vimos na Seção III, duas possíveis combinações de SM-NLMS seriam utilizando-se dois filtros com comprimentos ou $\gamma$ diferentes. Além de analisarmos aqui estes dois casos e suas possíveis aplicações, também estudaremos um método de cálculo eficiente de combinação convexa que aproveita a característica de atualizações esparsas do SM, além de uma variação deste, otimizada para cálculo de combinações de filtros com comprimentos diferentes.

As simulações desta seção foram feitas em condições similares às da Seção III, de forma que as SNR aqui mencionadas também se referem a um ruído branco gaussiano.

\section{A. Diferentes comprimentos}

Como vimos na Seção III, um filtro de comprimento menor tem maior velocidade de convergência que um de comprimento maior, que por sua vez apresenta um menor desajuste.

Simulamos a combinação convexa de dois SMF com comprimentos 64 e 256 , com valores de $\mu_{a}=100,1000$ e 10000, mantendo todos os demais parâmetros iguais: $S N R=$ $20 d B, \gamma=\sqrt{5} \sigma_{v}$ e $\mu_{S M}=1$. O resultado da simulação para $\mu_{a}=10000$ é apresentado na Fig. 5.

$\mathrm{O}$ filtro combinado funciona conforme esperado, reunindo a maior velocidade de convergência do filtro com $\mathrm{N}=64$ e o menor desajuste do filtro com $\mathrm{N}=256$. Constatamos que nas condições simuladas, o valor de $\mu_{a}$ que melhor conseguiu ajustar a combinação foi o de 10000 .
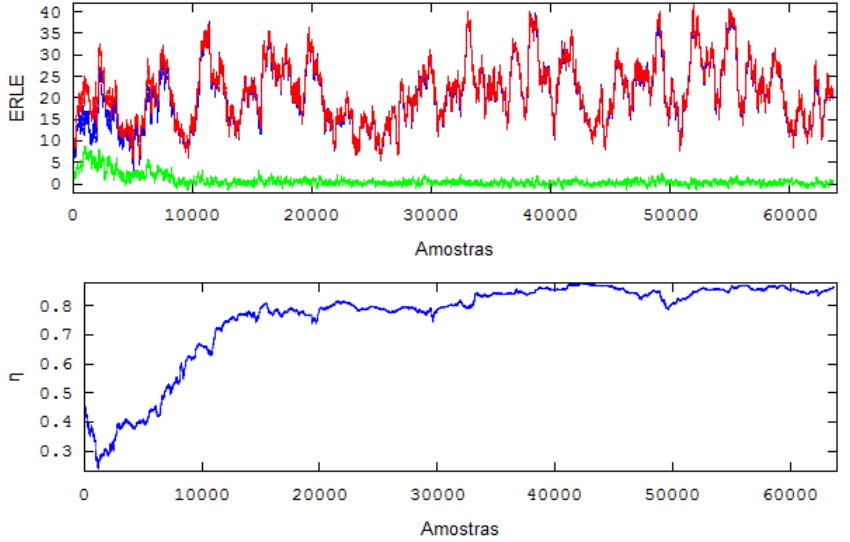

Fig. 5. (a) Combinação convexa de filtros SM-NLMS com $N=64$ e 256, $\mu_{a}=10000$ (vermelho), SM-NLMS simples com $\mathrm{N}=256$ (azul), diferença entre o primeiro e o segundo (verde). (b) $\eta(n)$ da combinação convexa.

\section{B. Diferentes $\gamma$}

A combinação de diferentes valores de $\gamma$ é útil basicamente em dois casos: 1) quando a SNR varia dentro de uma certa faixa conhecida ou é desconhecida, mas sabe-se que seu valor está compreendido nesta faixa; 2) quando a SNR varia abruptamente devido uma falha na detecção do DTD.

1) SNR assume valores dentro de uma faixa conhecida

Uma alternativa aos algoritmos de $\gamma$ variável é a combinação de filtros SM com $\gamma$ diferentes. Caso se conheça a faixa de valores que a SNR pode assumir e esta não seja muito larga, pode-se combinar filtros de $\gamma$ diferentes e tais que se tenha um bom desempenho em toda a faixa.

Suponhamos que numa certa aplicação, a SNR assuma valores entre 0 e $30 \mathrm{~dB}$. Vamos tentar escolher dois valores de $\gamma$ de modo a ter um desempenho razoável para SNR nesta faixa. Assim, lembrando que a Fig. 4 vale para várias SNR, podemos observar que para um dado $\gamma_{a}$ (que será ótimo para uma certa $S N R_{a}$ ), o ERLE médio do filtro não cai mais de $20 \%$ e a taxa de atualização não fica maior que $36 \%$ na faixa de $0,5 \gamma_{o}$ a $3 \gamma_{o}$, que equivale a uma variação de $-9,5 \mathrm{a}+6 \mathrm{~dB}$ no valor da $S N R_{a}$. Desta forma foram escolhidas as $S N R_{1}=9 d B$ e $S N R_{2}=24 d B$, que equivalem a $\gamma_{1}=0,00962$ e $\gamma_{2}=$ 0,00171 .
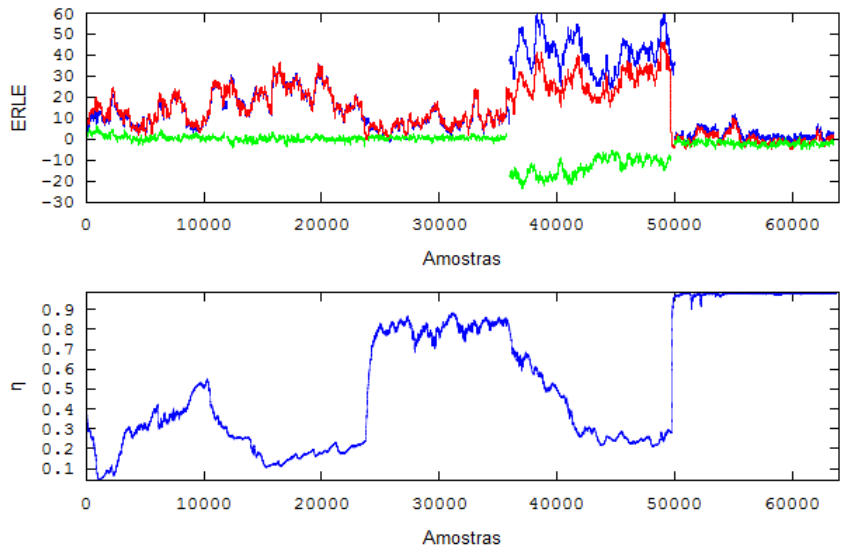

Fig. 6. Simulação do filtro SM-NLMS combinado submetido a várias SNR: $\mu_{a}=10000$. (a) resposta do filtro SM de $\gamma$ ideal para cada trecho (azul); resposta do filtro SM-NLMS combinado (vermelho) e diferença entre o primeiro e o segundo (verde). (b) $\eta(n)$ do filtro combinado. 
Para testar o desempenho da combinação, utilizamos o mesmo sinal de voz das simulações anteriores, adicionados de ruído branco gaussiano com as seguintes SNR: $15 \mathrm{~dB}$ no intervalo [1 12000], 20dB em [12001 24000], $10 \mathrm{~dB}$ em [24001 36000], 30dB em [36001 50000] e 0dB em [50001 64000].

A Fig. 6 mostra o resultado da simulação, onde se compara a resposta obtida pelo filtro combinado com a que um filtro SM de $\gamma$ ideal para cada trecho teria.

Nesta simulação vemos que, conforme esperado, o filtro combinado tende a escolher o SM de $\gamma$ menor nos intervalos onde a SNR é maior e vice versa. O filtro apresentou uma resposta muito próxima daquela do SMF de $\gamma$ ideal, com um ERLE inferior apenas no intervalo [36000 50000] devido a três fatores: o rastreamento dos filtros SM e o ajuste do fator de mistura da combinação (evidenciados no intervalo [36000 43000]) além do desajuste do SM devido ao $\gamma$ maior que o ótimo para a SNR em questão (evidenciado no intervalo [43000 50000]). O intervalo [43000 50000] também indica que no pior caso, após convergir, o ERLE médio obtido é da ordem de $20 \%$ abaixo do ótimo, como previsto nos cálculos de escolha dos $\gamma$.

Também foram feitas simulações para comparar o desempenho do filtro combinado com o SM simples (nas mesmas condições da simulação anterior). Nestas simulações comparamos o ERLE do filtro combinado $\left(E R_{c}\right)$ com o do SM simples, para três diferentes valores de $\gamma$, ótimos para as seguintes SNR: $0 \mathrm{~dB}\left(E R_{0}\right), 15 \mathrm{~dB}\left(E R_{15}\right)$ e $30 \mathrm{~dB}\left(E R_{30}\right)$. Como métrica de comparação utilizamos o $D(a, b)=\frac{\overline{\boldsymbol{a}(\boldsymbol{n})}-\overline{\boldsymbol{b}(\boldsymbol{n})}}{\overline{\boldsymbol{b}(\boldsymbol{n})}}$ (em que $a(n)$ e $b(n)$ são os valores médios dos respectivos sinais).

Os resultados, cujos gráficos foram omitidos por falta de espaço, foram: $D\left(E R_{0}, E R_{c}\right)=-59,0 \%, D\left(E R_{15}, E R_{c}\right)=$ $-22,4 \%$ e $D\left(E R_{30}, E R_{c}\right)=-19,4 \%$, ou seja, independentemente do $\gamma$ escolhido o SM simples apresentou um ERLE médio consideravelmente inferior ao da combinação.

Assim a combinação convexa de filtros SM se mostra como uma boa solução para o problema de escolha do parâmetro $\gamma$ em uma aplicação onde a SNR é ignorada ou variável, mas que tem seus valores dentro de uma faixa conhecida.

\section{2) Falha de detecção no DTD}

Quanto maior a SNR durante o double-talk, maior a probabilidade do DTD não detectar essa situação (e.g. [13]), permitindo que o filtro seja adaptado, o que fará seu desajuste aumentar. A combinação de dois SM tem a vantagem adicional de aumentar a robustez do algoritmo a erros do DTD.

Neste caso combina-se um SM com $\gamma$ baixo para a situação onde o DTD funciona corretamente (SNR maior) e outro com $\gamma$ elevado para a situação de falha do DTD (SNR menor). Desta forma, quando não houver falha de detecção a combinação escolherá a saída do SM de menor $\gamma$, caso contrário escolherá a saída do SM de maior $\gamma$.

Simulações preliminares indicaram que essa combinação apresenta um ERLE maior do que o de um filtro SM simples. Sendo assim interessante analisar melhor este tipo de aplicação em um estudo futuro.

\section{Cálculo eficiente da combinação convexa de SMF}

A característica de atualização seletiva de coeficientes do SM faz com que o seu cálculo seja feito de maneira esparsa, o que pode ser aproveitado para calcular alternadamente os filtros que compõem a combinação.

$\mathrm{O}$ algoritmo, que chamaremos de CECSM, basicamente funciona da seguinte forma: as saídas dos filtros F1 e F2 que compõem a combinação são calculadas continuamente (em todos os instantes de tempo $n$ ), assim como a saída da combinação e a atualização do seu parâmetro de mistura. Já o ajuste dos coeficientes dos filtros é feito de forma alternada, isto é, a cada instante de tempo $n$ apenas um dos filtros tem seus parâmetros atualizados. Caso um filtro não necessite de ajuste, o outro é atualizado (se necessário).

Para este algoritmo estudamos dois tipos de arbitragem na escolha de qual dos dois filtros terá seus parâmetros ajustados:

CECSM-I - ambos os filtros têm a mesma probabilidade de serem ajustados, isto é, se em dois instantes de tempo consecutivos ambos precisarem ser ajustados, no primeiro instante um será calculado e no segundo instante o outro o será, e assim por diante.

CECSM-II - um dos filtros tem prioridade, sendo que o outro só é ajustado quando este não precisar de ajuste. Ou seja, sempre que o filtro prioritário precisar de ajuste, ele será ajustado, enquanto que não-prioritário só será ajustado quando o prioritário não necessitar de ajuste.

A seguir discutiremos melhor os algoritmos, suas aplicações e sua complexidade computacional:

\section{1) CECSM-I}

Este algoritmo é útil para combinações onde os filtros têm o mesmo grau de prioridade, como no caso da combinação de filtros com diferentes $\gamma$. Para testar este algoritmo foi feita uma simulação com os coeficientes sendo atualizados continuamente e outra com eles sendo atualizados de forma alternada. Nestas simulações foram utilizados os seguintes parâmetros: $\gamma$ ótimo para o $S N R=20 \mathrm{~dB}, \quad N=256$ e $\mu_{S M}=1$.

Comparando os dois verificou-se que desempenho de ambos é muito semelhante, com o algoritmo alternado apresentando um ERLE médio até um pouco maior $(1,8 \%)$ que o do algoritmo contínuo.

\section{2) CECSM-II}

Este algoritmo é particularmente útil para a seguinte aplicação: combinam-se dois filtros de comprimentos diferentes, sendo que o menor tem seus coeficientes calculados de uma só vez e o maior tem seus coeficientes calculados em parcelas (como proposto em [8]).

Suponhamos o uso dos comprimentos 64 e 256: o filtro de comprimento $64\left(F_{64}\right)$ teria todos os seus coeficientes calculados no instante de tempo $n$; já o de comprimento 256 $\left(F_{256}\right)$ utilizaria, por exemplo, 4 iterações, tendo seus coeficientes calculados durante os instantes $n^{\prime}$ a $n^{\prime}+3$.

Caso utilizássemos o método de arbitragem CECSM-I, durante situações de rastreamento, o $F_{64}$ seria calculado apenas $20 \%$ do tempo, o que colocaria a perder sua qualidade de maior velocidade de convergência. Assim, considerando que o $F_{64}$ é importante apenas em situações de rastreamento e que fora dessa condição sua taxa de atualização é baixa, 
podemos utilizá-lo no CECSM-II como filtro prioritário, enquanto que o $F_{256}$, cuja importância é o menor desajuste, ficaria como não-prioritário.

Para testar o desempenho deste algoritmo foram feitas duas simulações utilizando a combinação dos filtros $F_{64}$ e $F_{256}$, onde os coeficientes do $F_{256}$ são calculados em grupos de 64 , em 4 iterações. Na primeira simulação utilizamos o CECSM-I e na segunda o CECSM-II, com o $F_{64}$ sendo o filtro prioritário. Os resultados são apresentados na Fig. 7, onde se vê que o CECSM-II realmente apresenta uma velocidade de convergência maior que a do CECSM-I, enquanto mantém o mesmo desajuste.

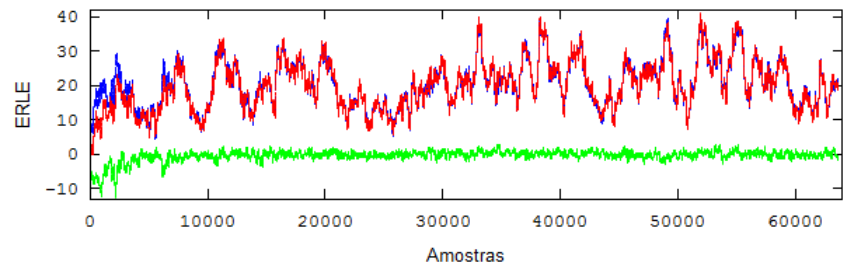

Fig. 7. Simulação dos algoritmos CECSM-II (azul), CECSM-I (vermelho) e diferença entre o primeiro e o segundo (verde). Parâmetros: $\gamma$ ótimo para o $S N R=20 \mathrm{~dB}, N_{1}=256, N_{2}=64$ e $\mu_{S M}=1$.

\section{3) Complexidade computacional}

Para comparar a complexidade computacional dos algoritmos, totalizamos o número de multiplicações, divisões e somas necessárias em cada um deles, no pior caso (ajuste constante dos coeficientes), considerando que o algoritmo proposto já tira vantagem da taxa de atualização do SM e que o hardware onde ele será implementado precise ser projetado para o pior caso.

Na Tabela 1 apresentamos os resultados tanto em função dos comprimentos $N_{1}$ e $N_{2} \quad\left(N_{1} \geq N_{2}\right)$ como em valores absolutos, para um caso de combinação de filtros de comprimentos diferentes e mesmo $\gamma$, sendo $N_{1}=256$ e $N_{2}=64$.

TABELA 1. COMPLEXIDADE COMPUTACIONAL

\begin{tabular}{|l|c|c|c|c|c|c|}
\cline { 2 - 7 } \multicolumn{1}{c|}{} & \multicolumn{3}{c|}{ Função de N } & \multicolumn{3}{c|}{ N1=256, N2=64 } \\
\hline \multicolumn{1}{c|}{ Algoritmo } & Mult. & Somas & Divis. & Mult. & Somas & Divis. \\
\hline \hline SM-NLMS & $3 \mathrm{~N}+1$ & $2 \mathrm{~N}+1$ & 1 & --- & --- & --- \\
\hline Comb.simples & $3 \mathrm{~N}_{1}+2 \mathrm{~N}_{2}+7$ & $2 \mathrm{~N}_{1}+\mathrm{N}_{2}+9$ & 4 & 903 & 581 & 4 \\
\hline CECSM-I & $3 \mathrm{~N}_{1}+\mathrm{N}_{2}+6$ & $2 \mathrm{~N}_{1}+7$ & 2 & 838 & 519 & 2 \\
\hline CECSM-II & $3 \mathrm{~N} 2+\mathrm{N} 1+6$ & $2 \mathrm{~N} 2+7$ & 2 & 454 & 135 & 2 \\
\hline
\end{tabular}

Destes resultados concluímos que o CECSM-I apresenta uma redução de complexidade razoável frente à combinação simples, com praticamente o mesmo desempenho. Já o CECSM-II apresenta uma redução substancial na complexidade, também com um desempenho semelhante. Porém o CECSM-II é destinado a uma aplicação específica, enquanto o CECSM-I tem um uso mais geral.

\section{CONCLUSÃO}

Neste artigo propusemos a combinação convexa de filtros SM-NLMS com diferentes parâmetros. Analisando quais diferentes parâmetros poderiam ser combinados, concluímos que combinando diferentes comprimentos poderíamos obter um filtro com boa relação entre velocidade de convergência e desajuste e que combinando diferentes $\gamma$ poderíamos ter um filtro SM menos susceptível a variações na SNR.

Estudando essas combinações e fazendo diversas simulações, constatamos que ambas as combinações propostas (diferentes valores de $\gamma$ e $\mathrm{N}$ ) apresentaram um bom desempenho em comparação com filtros SM simples. Particularmente a combinação com diferentes $\gamma$ se mostrou uma boa solução para o problema de definição do $\gamma$ nas aplicações em que se usa o SM.

Por fim, os algoritmos de cálculo eficiente de combinações SM-NLMS apresentaram, em comparação com o algoritmo simples, uma diminuição razoável na complexidade computacional no caso de combinação de filtros com o mesmo comprimento, e uma diminuição apreciável no caso de combinação de filtros com comprimentos diferentes e mesmo valor de $\gamma$.

\section{BIBLIOGRAFIA}

[1]. Gollamudi, Sridhar, et al. Set-Membership Filtering and a SetMembership Normalized LMS Algorithm with an Adaptive Step Size. IEEE SIGNAL PROCESSING LETTERS. May, 1998, Vol. 5. [2]. A Set-Membership NLMS Algorithm with Time-Varying Error Bound. Ganldino, Juraci F., Jr., José A. Apolinário e Campos, Marcello L. R. de. ISCAS : IEEE, 2006.

[3]. Arenas-Garcıa, Jeronimo, et al. Plant identification via adaptive combination of transversal filters. Elsevier. 2005. [4]. STEADY-STATE PERFORMANCE OF CONVEX COMBINATIONS OF ADAPTIVE FILTERS. Arenas-García, J.,

Figueiras-Vidal, A. R. e Sayed, A. H. IEEE : ICAASP, 2005. [5]. Zhang, Yonggang e Chambers, Jonathon A. Convex Combination of Adaptive Filters for a Variable Tap-Length LMS Algorithm. IEEE SIGNAL PROCESSING LETTERS. OCTOBER, 2006, Vol. 13, 10.

[6]. A Stable and Efficient DSP Implementation of a LSL Algorithm for Acoustic Echo Cancelling. Carezia, Andre H.C., et al. IEEE : ICAASP, 2001.

[7]. Breining et al. Acoustic Echo Control - An Application of VeryHigh Order Adaptive Filters. IEEE Signal Processing Magazine. July 1999.

[8]. On efficient implementations of the Set-Membership NLMS algorithm for real-time applications. Jose A. Apolinario Jr.,

Marcello L. R. de Campos. IEEE : TSI, 2006.

[9]. Homer, John, Bitmead, Robert R. e Mareels, Iven.

Quantifying the Effects of Dimension on the Convergence Rate of the LMS Adaptive FIR Estimator. IEEE TRANSACTIONS ON SIGNAL PROCESSING. October, 1998, Vol. 46.

[10]. Gu, Yuantao, et al. Convergence Analysis of a DeficientLength LMS Filter and Optimal-Length Sequence to Model Exponential Decay Impulse Response. IEEE SIGNAL PROCESSING LETTERS. January, 2003, Vol. 10.

[11]. Sayed, Ali H. Fundamentals of Adaptive Filtering. s.l. : John Wiley \& Sons, 2003.

[12]. SET-MEMBERSHIP ADAPTIVE FILTERING WITH PARAMETER-DEPENDENT ERROR BOUND TUNING. Guo, Li e Huang, Yih-Fang. IEEE : ICAASP, 2005.

[13]. Cho, Jun H., Morgan, Dennis R. e Benesty, Jacob. An Objective Technique for Evaluating Doubletalk Detectors in Acoustic Echo Cancelers. IEEE TRANSACTIONS ON SPEECH AND AUDIO PROCESSING. November, 1999, Vol. 7, 6. 